\title{
Ribotoxin genes in isolates of Aspergillus section Clavati
}

\author{
János Varga $\cdot$ Robert A. Samson
}

Received: 8 February 2008/ Accepted: 20 June 2008/Published online: 4 July 2008

(C) The Author(s) 2008

\begin{abstract}
Ribotoxins are ribosome inactivator proteins with high specificity against the sarcin/ricin domain of the $28 \mathrm{~S}$ ribosomal RNA. We examined the presence of ribotoxin genes in isolates of species recently assigned to Aspergillus section Clavati using specific primer pairs. All species assigned to this section have been found to carry ribotoxin genes. Phylogenetic analysis of the sequences of the amplified gene fragments allowed us to classify the genes to different groups including the $\alpha$-sarcin, gigantin, c-sarcin and mitogillin/restrictocin families. Two species, A. longivesica and N. acanthosporus produced ribotoxins which were only distantly related to gigantins and c-sarcins, respectively. Comparison of the protein sequences of the genes to known ribotoxin sequences revealed that all of them carry the presumed catalytic residues of ribotoxins, the cystein residues, and also the two Trp residues of $\alpha$-sarcin conserved in all ribotoxins known so far. These data indicate that these genes probably encode active ribotoxins. Further studies are in progress to examine the secretion and activities of these new ribotoxins.
\end{abstract}

J. Varga $(\bowtie) \cdot$ R. A. Samson

CBS Fungal Biodiversity Centre, Uppsalalaan 8,

3584 CT Utrecht, The Netherlands

e-mail: j.varga@cbs.knaw.nl

J. Varga

Department of Microbiology, Faculty of Science and Informatics, University of Szeged, 6701 Szeged,

P.O. Box 533, Hungary
Keywords Antifungal peptides - Aspergillus section Clavati · Clavin · Gigantin · Mitogillin · Phylogenetic analysis - Ribotoxins - Restrictocin

The ribotoxins are a family of ribosome inactivator proteins which have a highly specific activity against the phosphodiester bond of the sarcin/ricin domain universally preserved in 28S ribosomal RNA, leading to protein synthesis inhibition and cell death by apoptosis (Gasset et al. 1994; Kao et al. 2001; Kao and Davies 1995). Ribotoxins were discovered during a screening program searching for antibiotics and antitumor agents (Olson et al. 1963, 1965). The producing fungus was identified as Aspergillus giganteus MDH 18894, and the molecule responsible for these effects proved to be a protein, named $\alpha$-sarcin (Olson and Goerner 1965). Although ribotoxin production has also been observed in A. restrictus isolates NRRL 2869 (named restrictocin; Olson et al. 1963; Yang and Kenealy 1992; LópezOtín et al. 1984) and NRRL 3050 (named regulin; Olson and Goerner 1966), these isolates have later been re-identified as A. fumigatus (Summerbell 2002). Several other ribotoxins have been identified since then, including mitogillin (Rodriguez et al. 1982; Fernandez-Luna et al. 1985), clavin (Parente et al. 1996), gigantin (Salvarelli et al. 1994; Wirth et al. 1997), c-sarcin (Huang et al. 1997), and hirsutellins (Mazet and Vey 1995; Liu et al. 1995; Martínez-Ruiz et al. 1999b), a homolog of which have also been 
identified in the genomic DNA sequence of A. clavatus NRRL 1 (WC Nierman, unpublished; Genbank accession number XM_001273330). Aspf1, another ribotoxin produced by A. fumigatus has also been identified as a major allergen in Aspergillusrelated diseases (Arruda et al. 1992). This protein is secreted by the fungus and was detected in the urine of infected patients (Lamy et al. 1991). $\alpha$-Sarcin, restrictocin and Aspf1 are the most exhaustively characterized ribotoxins (Lacadena et al. 2007). These studies have indicated a high degree of conservation among ribotoxins, as those characterized display amino acid sequence similarities above $85 \%$, except for hirsutellins (Martínez-Ruiz et al. 1999b). Additionally, ribotoxin genes have also been identified ni Penicillium species (Hwu et al. 2001). This suggests that the presence of ribotoxins among fungi is more widespread than initially considered (Martínez-Ruiz et al. 1999a).

Ribotoxins have several advantages for use in the design of immunotoxins against cancer cells (Lacadena et al. 2007). However, their cytotoxic effects of ribotoxins hampered their use as therapeutic agents (Lacadena et al. 2007). Genetically engineered immunotoxins with increased stability and affinity are being developed (Rathore et al. 1997, Lacadena et al. 2007). Besides, identifying new types of ribotoxins could facilitate their application as agents against tumorigenic processes.

We examined the presence of ribotoxin genes in isolates of species accepted in Aspergillus section Clavati (Varga et al. 2007) using specific primer pairs. Phylogenetic analysis of the sequences of the amplified gene fragments has been carried out and the encoded protein sequences were compared to those available from public sequence databases.

The fungi examined included isolates belonging to all species allocated to Aspergillus section Clavati (Varga et al. 2007). The cultures were cultivated on malt peptone broth at $25 \mathrm{C}$ for 2 days, and DNA extractions have been carried out using the Masterpure $^{\mathrm{TM}}$ yeast DNA purification kit (Epicentre Biotechnol.) according to the instructions of the manufacturer. For PCR based detection of ribotoxins, the primer pair and PCR conditions developed by Lin et al. (1995) were applied as described previously (Varga et al. 2003). A DNA fragment of about 500 bp was amplified in some A. clavatus, A. giganteus, A. longivesica, A. clavatonanicus, A. rhizopodus and Neocarpenteles acanthosporus isolates. A. longivesica, N. acanthosporus and A. clavatonanicus have not been described previously as ribotoxin producers. In this and a previous study (Varga et al. 2003), we could amplify ribotoxin homologs from $80 \%$ of the examined isolates of Aspergillus section Clavati (24/30 isolates). A fragment of similar size have also been amplified in two isolates of Dichotomomyces cejpii, which has recently been accommodated in section Clavati based on phylogenetic analysis of various genomic loci (Varga et al. 2007; Peterson et al. 2008).

The amplified fragments have been subjected to sequence analysis. Sequence analysis was performed with the Big Dye Terminator Cycle Sequencing Ready Reaction Kit for both strands, and the sequences were aligned with the MT Navigator software (Applied Biosystems). The ribotoxin gene sequences have been deposited at the Genbank nucleotide sequence database under accession numbers EU308595-EU308610. Similarity searches in the Genbank database indicated that the D. cejpii sequences were related to a putative vanillin dehydrogenase sequence of A. clavatus NRRL 1, while all other sequences were similar to ribotoxin sequences of various fungi. None of the amplified ribotoxin gene fragments were found to carry introns (data not shown).

The sequence data were optimized using the software package Seqman from DNAStar Inc. Sequence alignments were performed by using CLUSTAL-X (Thompson et al. 1997) and improved manually. The neighbour-joining (NJ) method was used for the phylogenetic analysis. For NJ analysis, the data were first analysed using the Tamura-Nei parameter distance calculation model with gammadistributed substitution rates (Tamura and Nei 1993), which were then used to construct the $\mathrm{NJ}$ tree with MEGA version 3.1 (Kumar et al. 2004). To determine the support for each clade, a bootstrap analysis was performed with 1,000 replications. For parsimony analysis, the PAUP* version 4.0 software was used (Swofford 2000). Alignment gaps were treated as a fifth character state and all characters were unordered and of equal weight. Maximum parsimony analysis was performed for all data sets using the heuristic search option with 100 random taxa additions and tree bisection and reconstruction (TBR) as the branch-swapping algorithm. Branches of zero length 
were collapsed and all multiple, equally parsimonious trees were saved. The robustness of the trees obtained was evaluated by 1,000 bootstrap replications (Hillis and Bull 1993).

Phylogenetic analysis of the sequence data together with ribotoxin gene sequences collected from Genbank has been carried out using the neighbour-joining technique (Fig. 1). The topology of the resulting tree was the same as one of the 1,200 most parsimonious trees obtained by the PAUP software (106/321 parsimony informative characters; tree length: 212; consistency index: 0.7170 ; retention index: 0.9142). The ribotoxin gene sequences formed distinct clades on the tree, and were found to be related to either $\alpha$-sarcin, gigantin, $\mathrm{c}$-sarcin or mitogillin/restrictocins (Fig. 1). Most A. giganteus isolates produced ribotoxins related to gigantins, while A. clavatus CBS 106.45, A. clavatonanicus and the two A. rhizopodus isolates produced ribotoxins related to $\alpha$-sarcin. A. longivesica and $N$. acanthosporus produced ribotoxins which were only distantly related to gigantins and c-sarcins, respectively. None of the examined isolates assigned to Aspergillus section Clavati were found to produce ribotoxins belonging to either the c-sarcin or the mitogillin/restrictocin family (Fig. 1).
Regarding the value of ribotoxin sequences in a phylogenetic sense, the topology of the tree shown in Fig. 1 was compared to those obtained based on ITS and $\beta$-tubulin sequence data (Varga et al. 2007). The tree based on ribotoxin sequences could distinguish some of the species assigned to Aspergillus section Clavati (e.g. A. longivesica, N. acanthosporus and most $A$. giganteus isolates). However, ribotoxin genes of various $A$. clavatus isolates were found to belong to distinct clades, while those of A. clavatonanicus and $A$. rhizopodus were more closely related to A. clavatus than they were on trees based on other sequence data (data not shown; Varga et al. 2007). Interestingly, the $\alpha$-sarcin clade contained ribotoxin gene sequences from both Aspergilli and Penicillia (Fig. 1). Consequently, ribotoxin gene sequences cannot be used reliably for studying phylogenetic relationships among the producing species.

Comparison of the amino acid sequences of the ribotoxin genes of Aspergillus section Clavati to those of $\alpha$-sarcin, gigantin and mitogillin is presented in Fig. 2. The amino acid sequences of the ribotoxin genes examined were highly similar in agreement with previous findings (Lin et al. 1995; Lacadena et al. 2007). The amino acids found to be involved in proton transfer (His50, Glu96 and His137, labelled with *),
Fig. 1 Neighbour-joining tree based on partial nucleotide sequences of ribotoxin genes. The main families of fungal ribotoxins are indicated on the left. Numbers above branches represent bootstrap values; only values above $70 \%$ are shown. The ribotoxins produced by $N$. acanthosporus and $A$. longivesica isolates are labeled with $*$ and \#, respectively

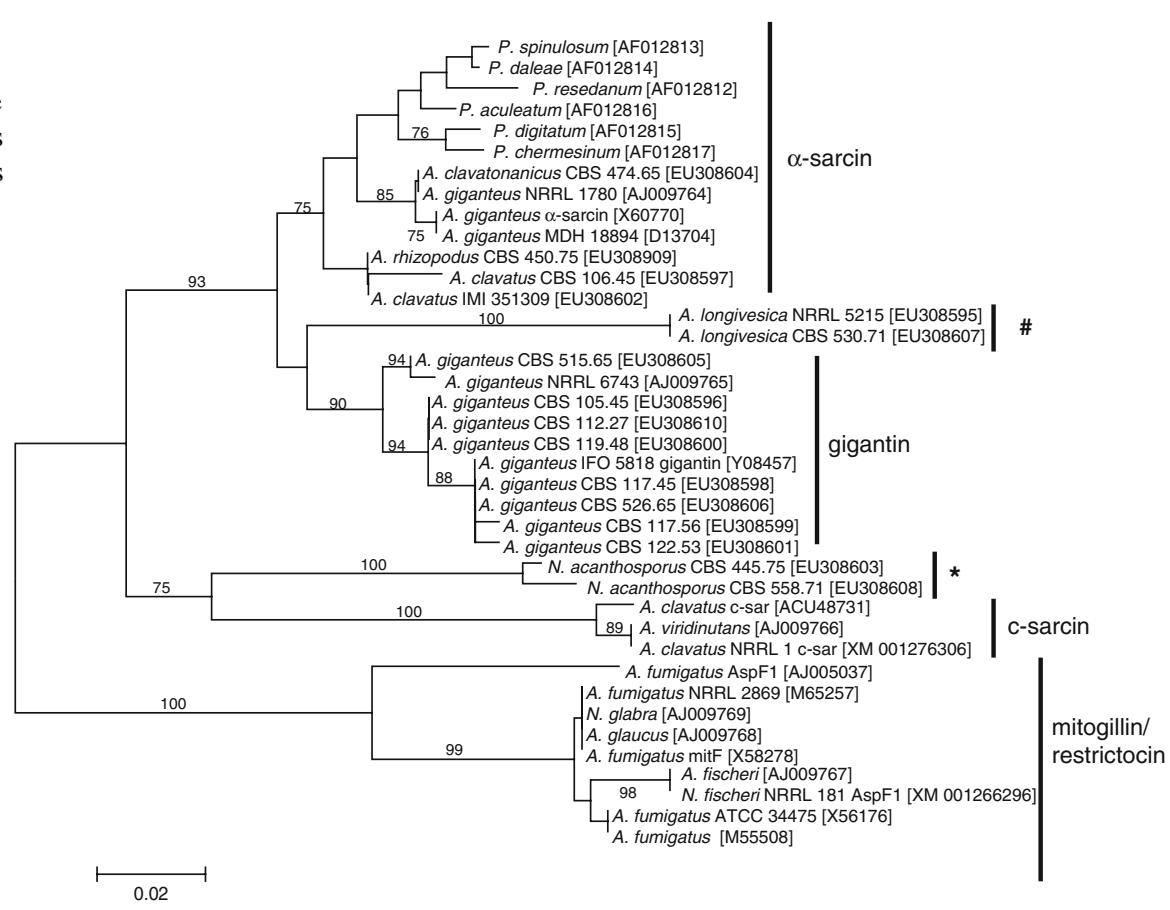




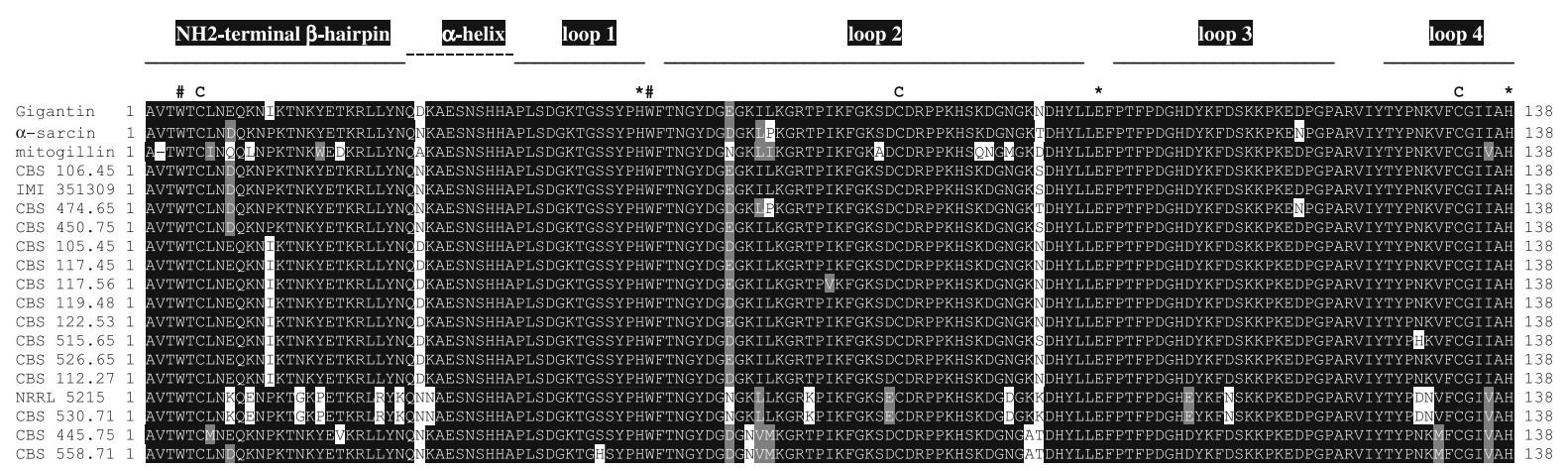

Fig. 2 Alignment of amino acid sequences of the ribotoxin genes identified. The amino acids found to be involved in proton transfer (His50, Glu96 and His137) are labelled with *, the two Trp residues of $\alpha$-sarcin are labelled with \#, while the

the two Trp residues of $\alpha$-sarcin conserved in all ribotoxins known so far (labelled with \#) and the Cys residues (labelled with $\mathrm{C}$ ) have been found in all sequences (Fig. 2; Lacadena et al. 2007). The ribotoxin gene sequences of A. longivesica and $N$. acanthosporus isolates differ from the other known ribotoxins in other, less conserved regions, indicating that these genes could encode for functional ribotoxins. However, further studies are needed to clarify if the ribotoxin genes of A. longivesica and $N$. acanthosporus encode functional ribosome-inactivating proteins.

In conclusion, we found that all species assigned to Aspergillus section Clavati carry ribotoxin genes, including A. longivesica, A. clavatonanicus and $N$. acanthosporus. Comparative analysis of the amino acid sequences of these gene fragments indicate that ribotoxins could be produced by these species. Further work is in progress to confirm the ribonucleolytic activities of cell-free extracts of these isolates, including those of A. longivesica and $N$. acanthosporus. Since these two species produce new types of ribotoxins, further detailed studies are needed to clarify their activities and their cytotoxic effects.

Open Access This article is distributed under the terms of the Creative Commons Attribution Noncommercial License which permits any noncommercial use, distribution, and reproduction in any medium, provided the original author(s) and source are credited.

\section{References}

Arruda LK, Mann BJ, Chapman MD (1992) Selective expression of a major allergen and cytotoxin, Aspf1, in Aspergillus fumigatus. Implications for the
Cys residues are labelled with $\mathrm{C}$. The main regions of the ribotoxin genes are shown above the alignment (Lacadena et al. 2007)

immunopathogenesis of Aspergillus-related diseases. J Immunol 149:3354-3359

Fernandez-Luna JL, López-Otin C, Soriano F, Méndez E (1985) Complete amino acid sequence of the Aspergillus cytotoxin mitogillin. Biochemistry 24:861-867

Gasset M, Mancheno JM, Lacadena J, Turnay J, Olmo N, Lizarbe MA, Martinez del Pozo A, Onaderra M, Gavilanes JG (1994) $\alpha$-Sarcin, a ribosome-inactivating protein that translocates across the membrane of phospholipid vesicles. Curr Topics Pept Protein Res 1:99-104

Hillis DM, Bull JJ (1993) An empirical test of bootstrapping as a method for assessing confidence in phylogenetic analysis. Syst Biol 42:182-192

Huang KC, Hwang YY, Hwu L, Lin A (1997) Characterization of a new ribotoxin gene (c-sar) from Aspergillus clavatus. Toxicon 35:383-392

Hwu L, Cho CJ, Tzeanz SS, Lin A (2001) Nucleotide sequence and the action of ribotoxin gene (sar gene) of Penicillium isolates from Taiwan. Bot Bull Acad Sin 42:101-107

Kao R, Davies J (1995) Fungal ribotoxins: a family of naturally engineered targeted toxins? Biochem Cell Biol 73: 1151-1159

Kao R, Martinez-Ruiz A, Martinez del Pozo A, Crameri R, Davies J (2001) Mitogillin and related fungal ribotoxins. Methods Enzymol 341:324-335

Kumar S, Tamura K, Nei M (2004) MEGA3: integrated software for molecular evolutionary genetics analysis and sequence alignment. Brief Bioinform 5:150-163

Lacadena J, Alvarez-Garcia A, Carreras-Sangra N, HerreroGalan E, Alegre-Cebollada J, Garcia-Ortega L, Onaderra M, Gavilanes JG, Martinez del Pozo A (2007) Fungal ribotoxins: molecular dissection of a family of natural killers. FEMS Microbiol Rev 31:212-237

Lamy B, Moutaouakil M, Latgé JP, Davies J (1991) Secretion of a potential virulence factor, a fungal ribonucleotoxin, during human aspergillosis infections. Mol Microbiol 5:1811-1815

Lin A, Huang K, Hwu L, Tzean SS (1995) Production of type II ribotoxins by Aspergillus species and related fungi in Taiwan. Toxicon 33:105-110

Liu WZ, Boucias DG, McCoy CW (1995) Extraction and characterization of the insecticidal toxin hirsutellin 
A produced by Hirsutella thompsonii var thompsonii. Exp Mycol 19:254-262

López-Otín C, Barber D, Fernández-Luna JL, Soriano F, Méndez E (1984) The primary structure of the cytotoxin restrictocin. Eur J Biochem 143:621-634

Martínez-Ruiz A, Kao R, Davies J, Martínez del Pozo A (1999a) Ribotoxins are a more widespread group of proteins within the filamentous fungi than previously believed. Toxicon 37:1549-1563

Martínez-Ruiz A, Martínez del Pozo A, Lacadena J, Oñaderra M, Gavilanes JG (1999b) Hirsutellin A displays significant homology to microbial extracellular ribonucleases. J Invertebr Pathol 74:96-97

Mazet I, Vey A (1995) Hirsutellin A, a toxic protein produced in vitro by Hirsutella thompsonii. Microbiology 141: $1343-1348$

Olson BH, Goerner GL (1965) Alpha-sarcin, a new antitumor agent. I. Isolation, purification, chemical composition, and the identity of a new amino acid. Appl Microbiol 13:314-321

Olson BH, Goerner GL (1966) Process for the production of regulin by Aspergillus restrictus and resulting product. US Patent 3,230,153

Olson BH, Harvey CI, Junek AJ, Jennings JC (1963) Restrictocin. US Patent 3,104,208

Olson BH, Jennings JC, Roga V, Junek AJ, Schuurmans DM (1965) a-Sarcin, a new antitumour agent. II. Fermentation and antitumour spectrum. Appl Microbiol 13:322-326

Parente D, Raucci G, Celano B, Pacilli A, Zanoni L, Canevari S, Adobati E, Colnaghi MI, Dosio F, Arpicco S, Cattel L, Mele A, De Santis R (1996) Clavin, a type-1 ribosomeinactivating protein from Aspergillus clavatus IFO 8605. cDNA isolation, heterologous expression, biochemical and biological characterization of the recombinant protein. Eur J Biochem 239:272-280

Peterson SW, Varga J, Frisvad JC, Samson RA (2008) Phylogeny and subgeneric taxonomy of Aspergillus. In: Varga J, Samson RA (eds) Aspergillus in the genomic era. Wageningen Academic Publishers, Wageningen, Netherlands, pp 33-56

Rathore D, Nayak SK, Batra JK (1997) Overproduction of fungal ribotoxin $\alpha$-sarcin in Escherichia coli: generation of an active immunotoxin. Gene 190:31-35
Rodriguez R, Lopez-Otin C, Barber D, Fernandez-Luna JL, Gonzalez G, Mendez E (1982) Amino acid sequence homologies in $\alpha$-sarcin, restrictocin and mitogillin. Biochem Biophys Res Commun 108:315-321

Salvarelli S, Muñoz S, Conde FP (1994) Purification and characterization of a ribonuclease from Aspergillus giganteus IFO 5818, the gigantin. Immunological and enzymic comparison with $\alpha$-sarcin. Eur J Biochem 225:243-251

Summerbell R (2002) Aspergillus, Fusarium, Sporothrix, Piedraia, and their relatives. In: Howard DH (ed) Pathogenic fungi in humans and animals. CRC Press, Boca Raton, Florida, pp 237-498

Swofford T (2000) PAUP*: phylogenetic analysis using parsimony. version 4.0. Sinauer Associates, Sunderland

Tamura K, Nei M (1993) Estimation of the number of nucleotide substitutions in the control region of mitochondrial DNA in humans and chimpanzees. Mol Biol Evol 10:512-526

Thompson JD, Gibson TJ, Plewniak F, Jeanmougin F, Higgins DG (1997) The ClustalX windows interface: flexible strategies for multiple sequence alignment aided by quality analysis tools. Nucleic Acids Res 25:4876-4882

Varga J, Due M, Frisvad JC, Samson RA (2007) Taxonomic revision of Aspergillus section Clavati based on molecular, morphological and physiological data. Stud Mycol 59:89-106

Varga J, Rigó K, Molnár J, Tóth B, Szencz S, Téren J, Kozakiewicz Z (2003) Mycotoxin production and evolutionary relationships among species of Aspergillus section Clavati. Antonie Van Leeuwenhoek 83:191-200

Wirth J, Martínez del Pozo A, Mancheño JM, Martínez-Ruiz A, Lacadena J, Oñaderra M, Gavilanes JG (1997) Sequence determination and molecular characterization of gigantin, a cytotoxic protein produced by the mould Aspergillus giganteus IFO 5818. Arch Biochem Biophys 343:188-193

Yang R, Kenealy WR (1992) Regulation of restrictocin production in Aspergillus restrictus. J Gen Microbiol 138:1421-1427 